\title{
The Neurotoxicity of Excitatory Amino Acids is Produced by Passive Chloride Influx ${ }^{1}$
}

\author{
STEVEN M. ROTHMAN²
}

Departments of Pediatrics, Neurology, and Anatomy and Neurobiology, Washington University School of Medicine, St. Louis, Missouri 63110

\begin{abstract}
In the $\mathbf{1 5}$ years since the neurotoxic properties of glutamate and related amino acids were first described, there has been no thoroughly convincing explanation of the pathophysiology of excitatory amino acid-induced neuronal death. These substances depolarize central neurons, increase the frequency of neuronal discharge, and augment synaptic activity, leading to the suggestion that one or more of these properties may in some way be responsible for toxicity. More recently, an excessive calcium influx triggered by amino acids has been implicated in this process.
\end{abstract}

As isolation of the different factors potentially involved in amino acid neurotoxicity is virtually impossible in vivo, dispersed hippocampal cultures were used to define the pathophysiology of this process in vitro. The toxicity of glutamate, $\mathrm{N}$-methyl-D-aspartate, and kainate was unaffected when calcium was deleted and tetrodotoxin added to the balanced salt solution bathing the cultures. In parallel experiments, the calcium ionophore A23187 was not toxic in the presence of calcium. These experiments failed to confirm a role for neuronal activity or calcium influx in this process. However, when depolarization was blocked by deleting sodium from the control salt solution, neither glutamate, $\boldsymbol{N}$-methyl-D-aspartate, nor kainate produced obvious changes. Alternately, when passive chloride influx was prevented by largely deleting chloride from the bath, the cells were also unchanged by the amino acids. Further experiments showed that depolarization produced by high external potassium concentrations or veratridine was also toxic, but only in the presence of external chloride.

These experiments suggest that the pathophysiology of amino acid neurotoxicity may be rather straightforward. These substances produce a steady depolarization which leads to an influx of chloride. Cations are then drawn into the neuron, which results in water entry and cell lysis.

Received June 18, 1984; Revised November 5, 1984;

Accepted November 8, 1984.

${ }^{1}$ This work was supported by National Institute of Neurological Communicative Disorders and Stroke Grants NS00568 and NS19988 and by the Epilepsy Foundation of America. I would like to thank Dr. Robert Miller for first suggesting the "chloride hypothesis," and Dr. John Olney for critiquing earlier drafts of the manuscript. I would also like to thank Dr. Kevin Martin, who provided calcium determinations; Mariam Samaie, who provided excellent technical help; and Aileen Derhake, who typed the paper.

${ }^{2}$ To whom all correspondence should be directed, at Department of Anatomy and Neurobiology, Washington University School of Medicine, 660 S. Euclid, St. Louis, MO 63110
In 1957, while studying hereditary retinal dystrophy in the mouse, Lucas and Newhouse (1957) serendipitously discovered that glutamate (GLU) injections produced rapid degeneration of the inner layer of the retina. Twelve years later, Olney (1969) described central nervous system lesions in mice after parenteral administration of GLU. Since then, the neurotoxic properties of GLU and a number of other dicarboxylic amino acids have been repeatedly documented (Olney 1978, 1983). Over this same time period, evidence has accumulated that GLU may be a major excitatory transmitter in the mammalian central nervous system (Roberts et al., 1981; Fonnum, 1984). In addition, GLU may be involved in a variety of human neurological diseases (Collins and Olney, 1982; Plaitakis et al., 1982; Shoulson, 1983). For these reasons, there has been a great deal of interest in the mechanism of GLU action, both as a putative neurotransmitter and as an established neurotoxin. In the mammalian central nervous system, GLU appears to open nondesensitizing channels which are permeable to at least sodium (Hablitz and Langmoen, 1982). This explains its well-established, prolonged depolarizing action.

The factors responsible for GLU neurotoxicity are not nearly so well known. Possibilities most often suggested include: (1) greatly increased frequency of neuronal activity (Olney, 1978); (2) sustained depolarization (Olney, 1978); and (3) excessive calcium influx from the extracellular space (Berdichevsky et al., 1983; Griffiths et al., 1983; Retz and Coyle, 1984). It is extremely difficult to investigate these hypotheses in intact animals. However, in vitro experiments utilizing cultures of dispersed fetal hippocampal neurons have demonstrated the ionic mechanism responsible for the neurotoxicity produced by GLU and the related excitatory amino acids $N$-methylD-aspartate (NMDA) and kainate (KA).

TABLE I

Composition of solutions

Solutions (mM)

\begin{tabular}{lrrrrrrr} 
& \multicolumn{8}{c}{ Solutions (mM) } \\
\cline { 2 - 8 } & A & B & C & D & E & F & G \\
\hline Sodium & 140 & 140 & & 140 & 55 & 54 & \\
Benzoylcholine & & & 140 & & & & \\
Potassium & 5 & 5 & 5 & 5 & 90 & 90 & 140 \\
Chloride & 172 & 172 & 172 & 32 & 172 & 21 & 21 \\
Tris $^{a}$ & 22 & 22 & 22 & 22 & 22 & 14 & 14 \\
Sulfate $_{\text {Glucose }}$ & 5.6 & 5.6 & 5.6 & 5.6 & 5.6 & 5.6 & 5.6 \\
Sucrose $^{b}$ & & & & 70 & & 85 & 85 \\
Calcium $_{\text {Magnesium }}$ & 0 & 0.5 & 0.5 & 0.5 & 0.5 & 0.5 & 0.5 \\
TIX & 5 & 4.5 & 4.5 & 4.5 & 4.5 & 4.5 & 4.5 \\
& $3 \times 10^{-3}$ & & & & & &
\end{tabular}

${ }^{a}$ Each $22 \mathrm{~mm}$ Tris contributes $17 \mathrm{~mm}$ chloride, which has been taken into account when calculating chloride concentration.

${ }^{b}$ Sucrose was used in solutions D, F, and G to maintain constant calculated osmolarity. 

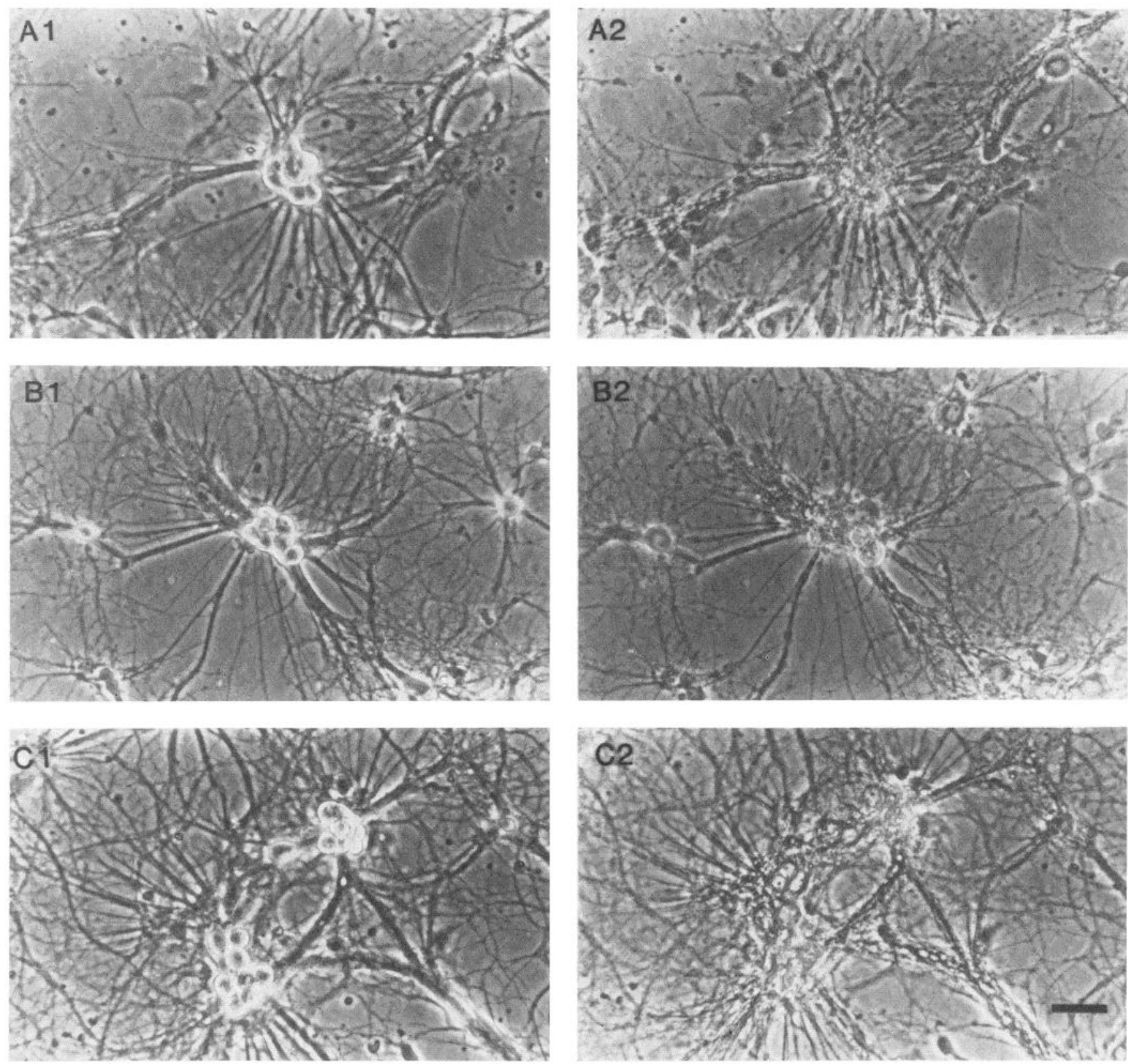

Figure 1. The composite photomicrograph shows individual microscope fields before ( $A 1$ to $C 1)$ and 30 min after cultures were exposed to GLU ( $A 2$ ), NMDA (B2), and KA (C2). Despite the addition of $\Pi \mathrm{TX}$ and removal of $\mathrm{Ca}$ from the bathing solution, the neurons all died after addition of the amino acids. Scale $=50 \mu \mathrm{m}$.

\section{Materials and Methods}

Culture. Dispersed cultures were made from hippocampi dissected from 18-d-old rat fetuses. The details of culture preparation were described in a previous report and have not been modified (Rothman, 1984). Hippocampal cultures used in these experiments were at least 2 weeks old.

Excitatory amino acid toxicity. In these experiments, individual microscope fields were photographed. The medium was then replaced with one of the balanced salt solutions (Table I, A, C, or D) which contained either $10^{-3} \mathrm{M}$ GLU, $10^{-4} \mathrm{M}$ NMDA, or $10^{-4} \mathrm{M}$ KA (all from Sigma). The same fields were then relocated and photographed after a 30-min incubation with the experimental solutions. Although only single fields are shown in the figures, they are representative of the appearance of the entire culture dish. Each experiment was repeated at least once, with identical results, for all the different solutions tested.

Solution A had tetrodotoxin (TTX) added to block regenerative sodium conductances. No calcium (Ca) was specifically added to this solution. The calcium concentration in four samples measured by atomic absorption spectroscopy prior to incubation with the cultures was $11.3 \pm 7.5 \mu \mathrm{M}$. After the 30 -min incubation, the calcium concentration in the same cultures increased to $116.3 \pm 40.5 \mu \mathrm{M}$, most likely due to leakage from the cultured cells. In solution $\mathrm{C}$, sodium $(\mathrm{Na})$ was totally replaced by benzoylcholine (Sigma). In solution D, all but $32 \mathrm{~mm}$ of chloride (Cl) was replaced with sulfate.

Calcium ionophore. The ionophore A23187 (Lilly, Indianapolis, IN) was freshly dissolved in dimethyl sulfoxide to make a $30 \mathrm{~mm}$ solution. This was diluted 1:10 to make a stock solution which was subsequently diluted with Tris-buffered saline (Table I, B) to a final concentration of $30 \mu \mathrm{M}$. The same microscope fields were photographed before and $30 \mathrm{~min}$ after exposure to this solution.

Veratridine depolarization. Veratridine (ICN Pharmaceuticals, Plainview, NY) was freshly dissolved in ethanol to make a $100 \mathrm{~mm}$ stock solution. This was then added to one of the balanced salt solutions (Table I, A, C, or D) to make a final concentration of $50 \mu \mathrm{M}$.

Potassium depolarization. Cultures were exposed to balanced salt solutions containing $90 \mathrm{~mm}$ potassium $(\mathrm{K})$ and $172 \mathrm{~mm} \mathrm{Cl}$ (Table I, E); $90 \mathrm{~mm} \mathrm{~K}$ 

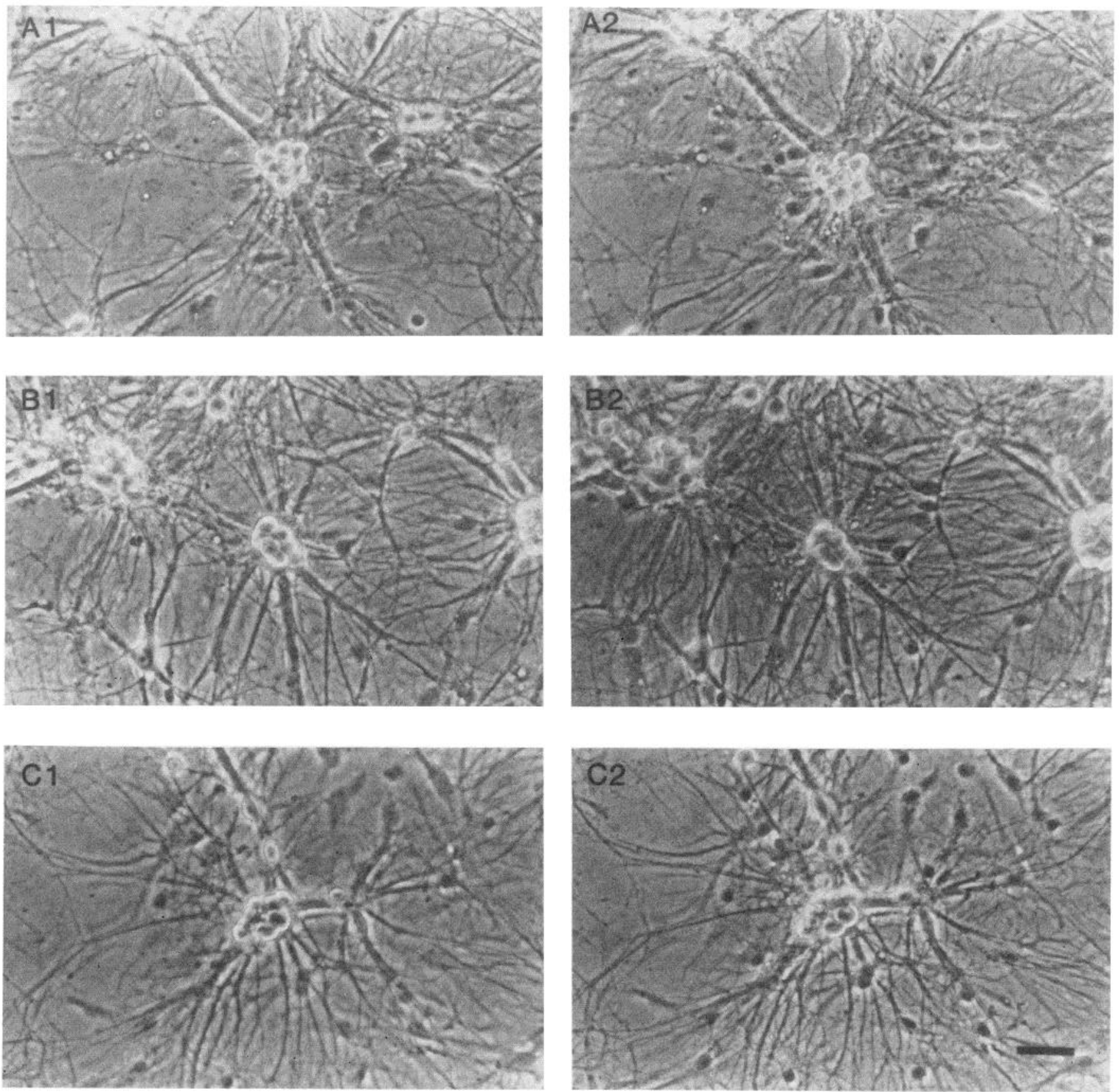

Figure 2. The composite photomicrograph shows sample fields prior to ( $A 1$ to $C 1$ ) and $30 \mathrm{~min}$ after exposure to GLU (A2), NMDA (B2), and KA (C2) The bulk of the $\mathrm{C} 1$ in the extracellular space was replaced with $\mathrm{SO}_{4}$ and neurons were virtually unchanged. Scale $=50 \mu \mathrm{m}$.

and only $21 \mathrm{~mm} \mathrm{Cl}$ (Table I, F); or $140 \mathrm{~mm} \mathrm{~K}$ and $21 \mathrm{~mm} \mathrm{Cl}$ (Table I, G). In the experiments with veratridine and high external $\mathrm{K}$ concentrations, identified fields were photographed before and after incubation with the experimental solution. All incubations were done in room air at $36^{\circ} \mathrm{C}$

Electrophysiology. For intracellular recording, culture medium was replaced with Hanks' balanced salt solution modified to contain $3 \mathrm{~mm} \mathrm{~K}, 5 \mathrm{~mm}$ $\mathrm{Ca}, 1 \mathrm{~mm}$ magnesium, $10 \mathrm{~mm}$ HEPES, and $3 \mu \mathrm{m}$ TTX. The culture was transferred to the stage of an inverted, phase-contrast microscope, which allowed cells to be impaled under direct vision. Microelectrodes were filled with $4 \mathrm{M}$ potassium acetate and had impedances of approximately 50 megohms. A standard high-impedance amplifier was used for recording.

GLU, NMDA, and KA were delivered by microperfusion (Choi and Fischbach, 1981). The amino acids were dissolved in medium identical to the bath and applied by pressure from blunt-tipped pipets $(\sim 5 \mu \mathrm{M})$ located approximately $30 \mu \mathrm{M}$ from the impaled neurons. The responses were photographed and measured with a digitizing tablet.

\section{Results}

Effects of decreased neuronal activity and low extracellular calcium. When neurons were exposed to $10^{-3} \mathrm{M}$ GLU (Fig. 1A), $10^{-4}$ $\mathrm{M}$ NMDA (Fig. $1 B$ ), or $10^{-4} \mathrm{M} \mathrm{KA}$ (Fig. $1 C$ ) in balanced salt solution (Table 1, A) which contained TTX and $5 \mathrm{~mm}$ magnesium and lacked added $\mathrm{Ca}$, they all died within $30 \mathrm{~min}$. There was disintegration of both cell bodies and processes, which was preceded in some cases by swelling of the soma. There was little difference between the effects of the three amino acids. In addition, the cultures deteriorated just as quickly as when they were exposed to excitatory amino acids in standard medium with normal extracellular Ca (Rothman, 1984). Thus, the neurotoxicity of GLU, NMDA, and KA does not depend on voltage-dependent $\mathrm{Na}$ channels or synaptic transmission.

As some $\mathrm{Ca}$ was still present in solution $\mathrm{A}$, it was still possible that $\mathrm{Ca}$ influx from the extracellular space was partly responsible for 

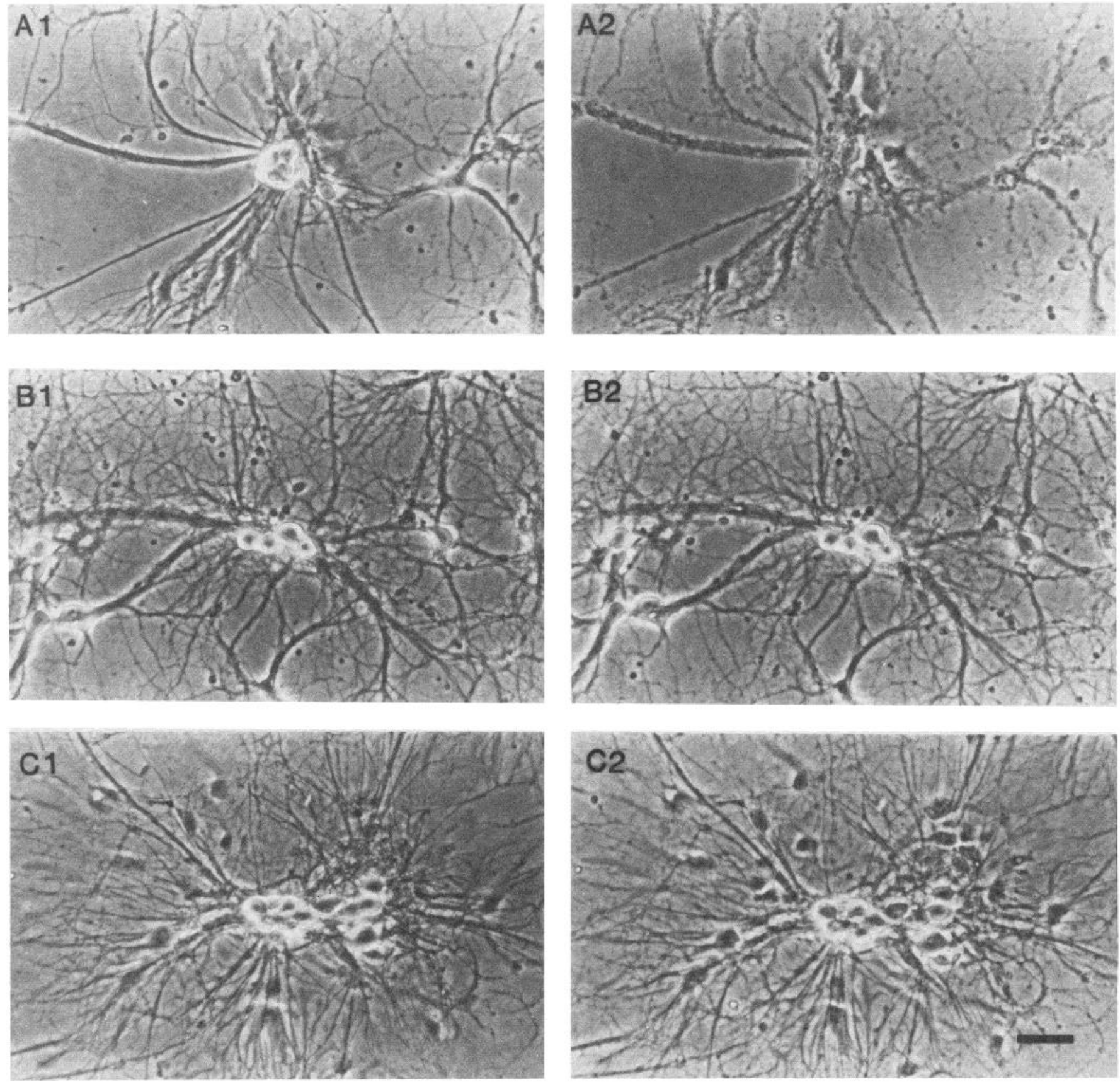

Figure 3. A1 shows a field before exposure to veratridine in a solution with no added Ca. After $30 \mathrm{~min}$, all neurons had been destroyed (A2). B1 shows a field in another culture prior to veratridine treatment in $\mathrm{Na}$-free salt solution. In the absence of $\mathrm{Na}$, neurons remained intact (B2). $\mathrm{Cl}$ shows a field prior to veratridine exposure in low $\mathrm{Cl}$ extracellular fluid. Under these conditions, neurons were unaffected by veratridine $(C 2)$. Scale $=50 \mu \mathrm{m}$.

the neuronal death. However, lowering the extracellular $\mathrm{Ca}$ further with EGTA was in itself toxic to the cultured neurons.

Effects of A23187. In an attempt to document that excessive $\mathrm{Ca}$ influx from the extracellular space could duplicate the toxic effects of excitatory amino acids, the cultures were exposed to $30 \mu \mathrm{M}$ A23187, a calcium ionophore (Schanne et al., 1979). In a solution containing $0.5 \mathrm{mM} \mathrm{Ca}$, the neurons were not noticeably changed after a 30-min incubation (not shown). As this same time period was sufficient for the neurotoxic amino acids to produce total destruction of all neurons in a solution containing far less extracellular $\mathrm{Ca}$, it appeared unlikely that this process was mediated by $\mathrm{Ca}$ influx.

$\mathrm{Na}$ substitution. Although regenerative $\mathrm{Na}$ channels blocked by TTX are unnecessary for amino acid neurotoxicity, depolarization or $\mathrm{Na}$ influx might still be important. Accordingly, benzoylcholine was substituted for $\mathrm{Na}$ (Table I, C) to block the depolarization produced by the amino acids, which is likely due to $\mathrm{Na}$ influx (Hablitz and Langmoen, 1982). At the same time, the cultures were exposed to $10^{-3} \mathrm{M} \mathrm{GLU}, 10^{-4} \mathrm{M}$ NMDA, and $10^{-4} \mathrm{M} \mathrm{KA}$. Under these conditions, even with $\mathrm{Ca}$ in the bath, the neurons were not visibly changed after $30 \mathrm{~min}$. These experiments suggest a role for $\mathrm{Na}$ influx and/or depolarization in producing amino acid neurotoxicity. However, they do not allow the two variables to be separated.

$\mathrm{Cl}$ substitution. Steady depolarization of neurons produces a $\mathrm{Cl}$ influx because of the altered electrochemical gradient for $\mathrm{Cl}$. It therefore seemed possible that $\mathrm{Cl}$ influx, eventually resulting in osmotic lysis, might account for the observed neurotoxicity of the amino acids (Miller and Slaughter, 1985). To test this hypothesis, an impermeant anion, sulfate $\left(\mathrm{SO}_{4}\right)$, was substituted for the bulk of the 

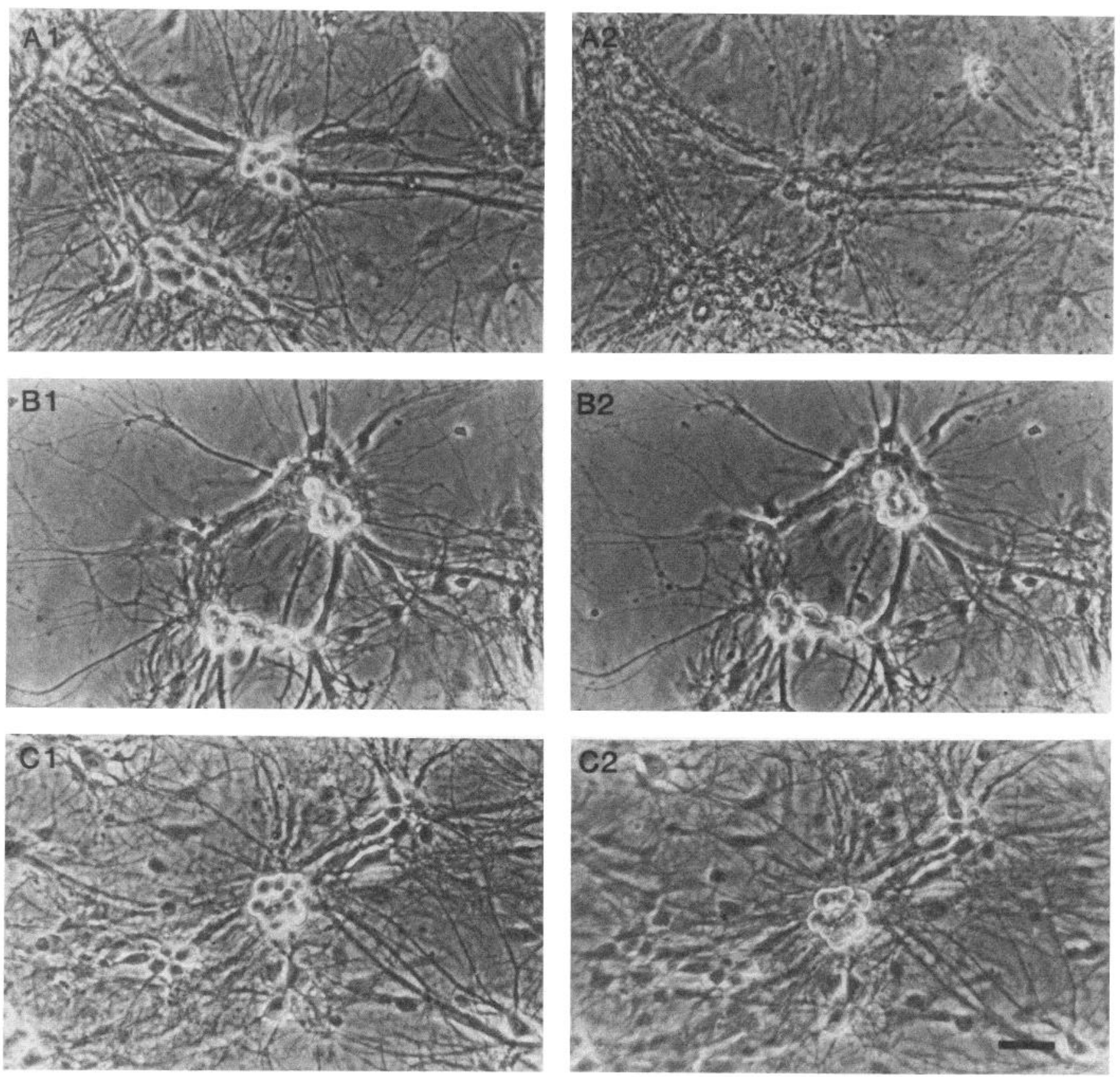

Figure 4. A shows a field before $(A 1)$ and $30 \mathrm{~min}$ after $(A 2)$ exposure to $90 \mathrm{~mm} \mathrm{KCl}$ in balanced salt solution. All neurons were destroyed by this treatment. $B$ shows another field before $(B 1)$ and $30 \mathrm{~min}$ after $(B 2)$ exposure to $90 \mathrm{~mm} \mathrm{~K}$ in low $\mathrm{Cl}$ solution. The cells were unchanged. $C$ shows a field before (C1) and after (C2) treatment with $140 \mathrm{~mm} \mathrm{~K}$ in low Cl solution. Again, neurons were unaffected. Scale $=50 \mu \mathrm{m}$.

extracellular $\mathrm{Cl}$ in the bath (Table I, D). GLU, NMDA, and KA were then added at the same concentrations previously used. After a 30 min exposure, the neurons were virtually unchanged (Fig. 2, A to C), implying that $\mathrm{Cl}$ influx is the critical factor for the production of amino acid neurotoxicity. Similar results were obtained when $\mathrm{Cl}$ was replaced by isethionate.

Veratridine depolarization. If the mechanism suggested above is correct, then other depolarizing agents should also be neurotoxic, and this toxicity should be blocked by chloride removal. Veratridine, which opens voltage-dependent $\mathrm{Na}$ channels (Ulbricht, 1969) should, therefore, be toxic. When neurons were treated with $50 \mu \mathrm{M}$ veratridine in medium deficient in $\mathrm{Ca}$ (solution $\mathrm{A}$ without TTX), they were all destroyed after $30 \mathrm{~min}$ (Fig. 3A). Replacement of $\mathrm{Na}$ by benzoylcholine (Table I, C) protected the neurons from the same veratridine concentration (Fig. $3 B$ ). Finally, replacement of the bulk of the $\mathrm{Cl}$ by $\mathrm{SO}_{4}$ retaining the normal extracellular $\mathrm{Na}$ concentration (Table I, D) also blocked veratridine toxicity, consistent with the hypothesis that passive $\mathrm{Cl}$ entry mediates this type of neuronal death (Fig. 3C).

Potassium depolarization. One other paradigm was used to acquire more evidence that passive chloride influx associated with depolarization and not depolarization per se, was responsible for the neuronal destruction observed in these experiments. Cultures were depolarized with a balanced salt solution in which $90 \mathrm{mM} \mathrm{Na}$ was replaced by an equivalent amount of $\mathrm{K}$ (Table I, E). The neurons in these cultures all disintegrated within $30 \mathrm{~min}$ and appeared identical to neurons treated with one of the neurotoxic amino acids (Fig. 4A). However, cultures exposed to an identical $\mathrm{K}$ solution in which $\mathrm{SO}_{4}$ replaced the bulk of the $\mathrm{Cl}$ (Table I, F) were unchanged after $30 \mathrm{~min}$ (Fig. $4 B$ ). In fact, even when cultures were incubated 
A
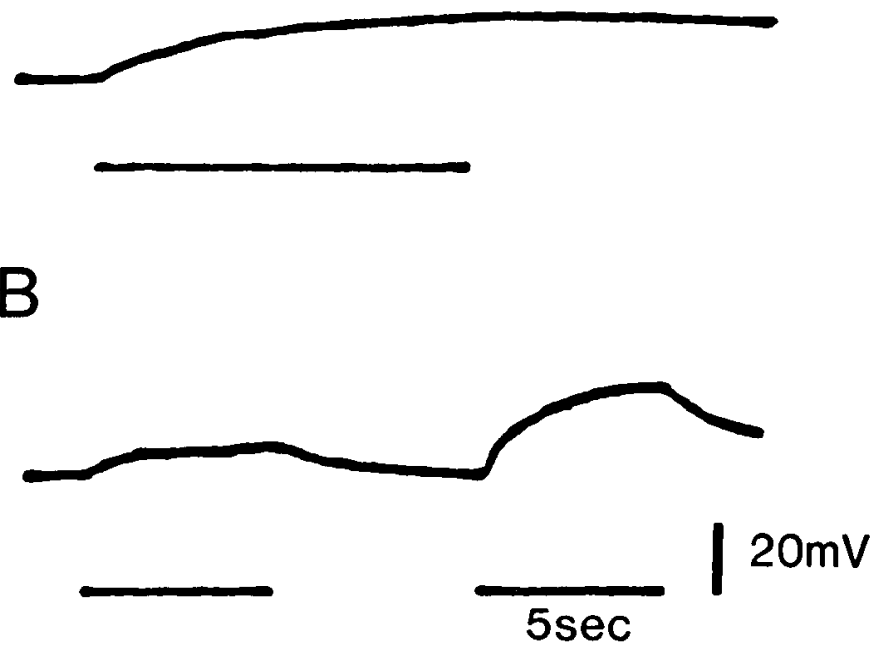

Figure 5. Effects of amino acid microperfusion. In $A, 10^{-3} \mathrm{M}$ GLU applied for $10 \mathrm{sec}$ (marker) produced a steady depolarization which did not desensitize. In $B, 10^{-4} \mathrm{M}$ NMDA (first marker) and $10^{-4} \mathrm{M} \mathrm{KA}$ (second marker) were sequentially applied near another neuron. Both amino acids always produced steady depolarizations. Resting membrane potential was $65 \mathrm{mV}$ for both $A$ and $B$.

TABLE ॥

Amino acid depolarization

Average Resting Potential Average Depolarization from of Neurons Tested $(\mathrm{mV}) \quad$ Resting Level $(\mathrm{mV})$

\begin{tabular}{lcc}
\hline GLU $(n=7)$ & $57 \pm 6$ & $29 \pm 13$ \\
NMDA $(n=13)$ & $69 \pm 9$ & $6 \pm 5$ \\
KA $(n=6)$ & $68 \pm 9$ & $20 \pm 7$ \\
\hline
\end{tabular}

with $140 \mathrm{mM} \mathrm{K}$ in a low-Cl salt solution (Table I, G), they showed virtually no morphological changes when viewed under phase-contrast optics (Fig. 4C). This set of experiments provided more evidence in support of the hypothesis that neurons can tolerate prolonged depolarization, so long as they are not exposed to a normal $\mathrm{Cl}$ concentration

Physiological effects of neurotoxic amino acids. The pathophysiology of amino acid neurotoxicity proposed above depends upon their ability to produce prolonged, nondesensitizing depolarizations. Accordingly, responses to GLU, NMDA, and KA were monitored with intracellular recording. No attempt was made to differentiate responses to the different amino acids by polarizing the resting membrane potential (Dingledine, 1983). Prolonged (5 to $10 \mathrm{sec}$ ) applications of all three amino acids did result in steady depolarizations which did not decrease over time (Fig. 5). GLU produced the greatest depolarization, then KA, and then NMDA (Table II). The differences in magnitude of the depolarizations could not be accounted for by depolarization of resting membrane potential, as cells tested with GLU tended to have lower resting potentials than those tested with the other two amino acids (Table II).

\section{Discussion}

The experiments described above suggest that the pathophysiology of amino acid neurotoxicity may be rather straightforward. $\mathrm{Cl}$ is predominantly passively distributed across the neuronal membrane, such that the ratio of intracellular to extracellular $\mathrm{Cl}$ can be calculated from the Nernst relationship:

$$
E m \simeq 58 \log \frac{[\mathrm{Cl} \text { in }]}{[\mathrm{Cl} \text { out }]}
$$

where Em is the neuron's resting potential. Depolarization increases the intracellular $\mathrm{Cl}$ concentration, which cannot be compensated by outward anion flux, because the bulk of intracellular anions are impermeant. Therefore, more cations are drawn into the cell, which increases intracellular osmolarity. This leads to water entry (Kuffler et al., 1984). Eventually, tension on the cell membrane increases and the neuron lyses. This scheme is not unique for the excitatory amino acids, as high external potassium concentrations and veratridine appear to produce neuronal death by the same mechanism. Elimination of $\mathrm{Na}$ protects the cells from amino acids and veratridine because it prevents the initial depolarization. Removing most of the extracellular $\mathrm{Cl}$ decreases $\mathrm{Cl}$ flux into the neurons and allows them to tolerate depolarization without accumulating an excessive osmotic load.

No evidence was found to support recent suggestions that $\mathrm{Ca}$ influx is responsible for amino acid toxicity (Berdichevsky et al., 1983; Griffiths et al., 1983; Retz and Coyle, 1984). Removal of the bulk of extracellular Ca did not visibly alter killing by any of the amino acids. Exposure to the Ca ionophore A23187 also had no immediate effect on the cultured neurons despite its ability to kill hepatocytes in vitro (Schanne et al., 1979). While the neurotoxic amino acids may cause $\mathrm{Ca}$ influx, this does not play a role in the pathophysiology of neuronal death reported here. lon substitution experiments have convincingly shown that excessive $\mathrm{Ca}$ influx plays a role in the degeneration at the mammalian neuromuscular junction produced by prolonged exposure to cholinomimetics (Leonard and Salpeter, 1979). Recent observations at the locust neuromuscular junction have also indicated that elevated intracellular Ca may be responsible for degeneration produccd by GLU, the putative neurotransmitter at this site (Donaldson et al., 1983). Therefore, postsynaptic degeneration at the neuromuscular junction is most likely a very different process from amino acid neurotoxicity and is probably not an appropriate model system for studying the latter.

Synaptic release of other neurotransmitters and regenerative $\mathrm{Na}$ potentials also appear unnecessary for full expression of amino acid neurotoxicity. All three amino acids were toxic in medium containing $5 \mathrm{~mm}$ magnesium, almost no $\mathrm{Ca}$, and $\mathrm{TTX}$, which would very effectively prevent transmitter release and $\mathrm{Na}$ spikes. It might not be expected that KA would be directly toxic, as a number of in vivo studies have demonstrated a requirement for intact innervation and presumably transmitter release in regions susceptible to KA toxicity. The optic tectum, striatum, and dentate region of hippocampus are all protected from KA lesions if the appropriate input pathways are lesioned (Streit et al., 1980; Biziere and Coyle, 1978; Kohler et al., 1978). Other studies have suggested that electrical seizures triggered by KA, and not KA per se, are responsible for brain damage (Nadler and Cuthbertson, 1980; Sloviter and Damiano, 1981). While there is no definite explanation for the discrepancy between the in vivo and in vitro results, it is certainly possible that release of intrinsic neurotransmitter and/or epileptiform activity potentiates the toxicity of low doses of KA. However, the present study certainly shows that KA at concentrations of $100 \mu \mathrm{M}$, and possibly lower, can be directly toxic to neurons.

One other initially puzzling result was the ability of NMDA to produce toxicity identical to that of GLU and KA, despite its slight depolarizing effect. Application of $10^{-4} \mathrm{M}$ NMDA for periods of seconds depolarized cells an average of $6 \mathrm{mV}$ from resting level (Table II). This was far more hyperpolarized than the threshold resting potential for $\mathrm{KCl}$-induced neuronal death, which was estimated to be $-16 \mathrm{mV}$. The explanation for this apparent discrepancy is that

${ }^{3}$ Rapid $(30 \mathrm{~min}) \mathrm{KCl}$ toxicity was seen with $[\mathrm{KCl}] \geqslant 75 \mathrm{~mm}$. Assuming that normal intracellular K is approximately $140 \mathrm{~mm}$ (Katzman and Pappius, 1973), and that the neuron behaves as a $K$ electrode, the resting potential in $75 \mathrm{~mm}$ $K$ can be calculated from the Nernst relationship:

$$
E m \simeq E_{k}=-58 \log \frac{[\mathrm{K} \text { in }]}{[\mathrm{K} \text { out }]}=-16 \mathrm{mV}
$$


the nondesensitizing depolarization produced by NMDA is the result of an inward $\mathrm{Na}$ current which causes an equal but outward $\mathrm{K}$ current. Over a number of minutes, the transmembrane $K$ gradient will gradually decrease, resulting in depolarization until the cell reaches threshold for osmotic lysis.

The observations on NMDA toxicity may also have implications for the physiological basis of NMDA action. Hippocampal pyramidal neurons maintained in the slice produce slow, regenerative spikes following NMDA iontophoresis (Dingledine, 1983). These spikes have properties similar to $\mathrm{Ca}$ action potentials, but might also be produced by $\mathrm{Na}$ influx. That NMDA toxicity was prevented by substituting benzoylcholine for $\mathrm{Na}$ demonstrates that the channel opened by NMDA does allow Na passage.

Ihese studies on amino acid neurotoxicity may eventually prove relevant to human neurobiology. The synaptic release of GLU, or a closely related amino acid, appears to be responsible for hypoxic neuronal injury. When cultured neurons were pretreated with $\gamma$-Dglutamylglycine, a postsynaptic blocker of excitatory amino acids, they were remarkably resistant to anoxia (Rothman, 1984). GLU release may contribute to brain damage in regions of uncontrolled epileptic activity (Collins and Oney, 1982). The neuropathological lesions found in patients with Huntington's disease suggest that the abnormal gene codes for a defect which predisposes the carrier to the neurotoxicity of GLU (Shoulson, 1983). Finally, a partial deficiency of the enzyme glutamate dehydrogenase in patients with olivopontocerebellar-atrophy may allow high concentrations of GLU to accumulate in the brain. This has been postulated to produce the neuronal degeneration seen in that disorder (Plaitakis et al., 1982). Should a role for GLU, or a related amino acid, be confirmed in the pathogenesis of any of these disorders, knowledge of its mechanism of action will aid in the design of rational therapy.

\section{References}

Berdichevsky, E., N. Riveros, S. Sanchez-Armass, and F. Orrego (1983) Kainate, $N$-methylaspartate and other excitatory amino acids increase calcium influx into rat brain cortex cells in vitro. Neurosci. Lett. 36: 75-80.

Biziere, K., and J. Coyle (1978) Influence of cortico-striatal afferents on striatal kainic acid neurotoxicity. Neurosci. Lett. 8: 303-310.

Choi, D. W., and G. Fischbach (1981) GABA conductance of chick spinal cord and dorsal root ganglion neurons in cell culture. J. Neurophysiol. 45: 605-620.

Collins, R. C., and J. Olney (1982) Focal cortical seizures cause distant thalamic lesions. Science 218: 177-179.

Dingledine, R. (1983) N-Methylaspartate activates voltage dependent calcium conductance in rat hippocampal pyramidal cells. J. Physiol. (Lond.) 343: 385-405.

Donaldson, P. L., I. Duce, and P. Usherwood (1983) Calcium accumulation precedes the degenerative effects of L-glutamate on locust muscle fibre. Brain Res. 274: 261-265.

Fonnum, F. (1984) Glutamate: A neurotransmitter in mammalian brain. J. Neurochem, 42: 1-11.
Griffiths, T., M. C. Evans, and B. S. Meldrum (1983) Temporal lobe epilepsy, excitotoxins and the mechanism of selective neuronal loss. In Excitotoxins, K. Fuxe, P. Roberts, and R. Schwarcz, eds., pp. 331-342, MacMillan, London.

Hablitz, J., and I. Langmoen (1982) Excitation of hippocampal pyramidal cells by glutamate in the guinea pig and rat. J. Physiol. (Lond.) 325: 317331.

Katzman, R., and H. Pappius (1973) Brain Electrolytes and Fluid Metabolism, pp. 114-115 and 184-186, Williams and Wilkins, Baltimore.

Kohler, C., R. Schwarcz, and K. Fuxe (1978) Perforant path transections protect hippocampal granule cells from kainate lesion. Neurosci. Lett. 10. 241-246.

Kuffler, S. W., J. G. Nicholls, and A. R. Martin (1984) From Neuron to Brain, pp. 111-118, Sinauer, Sunderland, MA.

Leonard, J. P., and M. Salpeter (1979) Agonist-induced myopathy at the neuromuscular junction is mediated by calcium. J. Cell. Biol. 82: 811-819.

Lucas, D. R., and J. P. Newhouse (1957) The toxic effect of sodium L-glutamate on the inner layers of the retina. Arch. Ophthalmol. 58: 193201.

Miller, R. F., and M. M. Slaughter (1985) Excitatory amino acid receptors in the vertebrate retina. In Retinal Transmitters and Modulators: Models for the Brain, W. W. Morgan, ed., CRC Press, Boca Raton, in press.

Nadler, J. V., and G. J. Cuthbertson (1980) Kainic acid neurotoxicity toward hippocampal formation: Dependence on specific excitatory pathways. Brain Res. 195: 47-56.

Olney, J. W. (1969) Brain lesions, obesity and other disturbances in mice treated with monosodium glutamate. Science 164: 719-721.

Olney, J. W. (1978) Neurotoxicity of excitatory amino acids. In Kainic Acid as a Tool in Neurobiology, E. McGeer, J. W. Olney, and P. L. McGeer, eds., pp. 95-121, Raven Press, New York.

Olney, J. W. (1983) Excitotoxins: An Overview. In Excitotoxins, K. Fuxe, P. Roberts, and R. Schwarcz, cds., pp. 82-96, MacMillan, London.

Plaitakis, A. S., S. Berl, and M. Yahr (1982) Abnormal glutamate metabolism in an adult-onset degenerative neurological disorder. Science 216: 193196.

Retz, K. C., and J. Coyle (1984) The differential effects of excitatory amino acids on uptake of ${ }^{45} \mathrm{CaCl}_{2}$ by slices from mouse striatum. Neuropharmacology 23: 89-94.

Roberts, P., J. Storm-Mathisen, and G. Johnston, eds. (1981) Glutamate. Transmitter in the Central Nenous System, Wiley-Interscience, New York.

Rothman, S. M. (1984) Synaptic release of excitatory amino acid neurotransmitter mediates anoxic neuronal death. J. Neurosci. 4: 1884-1891.

Schanne, F. A., A. Kane, E. Young, and J. Farber (1979) Calcium dependence of toxic cell death: a final common pathway. Science 206: 699-700.

Shoulson, I. (1983) Huntington's disease: anti-neurotoxic therapeutic strategies. In Excitotoxins, K. Fuxe, P. Roberts, and R. Schwarcz, eds., pp. 343-353, MacMillan, London.

Sloviter, R. S., and B. P. Damiano (1981) On the relationship between kainic acid-induced epileptiform activity and hippocampal neuronal damage. Neuropharmacology 20: 1003-1011.

Streit, P., M. Stella, and M. Cuenod (1980) Kainate-induced lesion in the optic tectum: Dependency upon optic nerve afferents or glutamate. Brain Res. 187: $47-57$.

Ulbricht, W. (1969) The effect of veratridine on excitable membranes of nerve and muscle. Ergeb. Physiol. Biol. Chem. Exp. Pharmacol. 61: 18-71. 